\title{
On the Performance Analysis of Multirelay Cooperative Diversity Systems With Channel Estimation Errors
}

\author{
Osama Amin, Student Member, IEEE, Salama Said Ikki, Member, IEEE, and Murat Uysal, Senior Member, IEEE
}

\begin{abstract}
In this paper, we investigate the performance of an amplify-and-forward (AF) cooperative diversity system with multiple relays in the presence of channel estimation errors. We consider both conventional relaying (in which all relay nodes participate in the relaying phase) and opportunistic relaying (in which only a single relay is allowed to participate). We derive closed-form expressions for error probability, outage probability, and ergodic channel capacity. The derivations are confirmed through Monte Carlo simulations. We further deploy the derived expressions to obtain optimal power allocation rules for performance improvements.
\end{abstract}

Index Terms-Amplify-and-forward (AF) relaying, channel estimation, cooperative diversity, fading channels, performance analysis.

\section{INTRODUCTION}

$\mathbf{C}$ OOPERATIVE diversity has emerged as a powerful technique to realize spatial diversity advantages in a distributed manner [1], [2]. Taking advantage of the broadcast nature of the radio-frequency transmission, cooperative diversity creates a virtual antenna among the nodes that are willing to share their resources. In the literature, various cooperation protocols, coupled with different relaying modes, have been proposed [3], [4]. A commonly employed form of cooperation protocol is the so-called receive diversity (RD) protocol, which is also known as orthogonal relaying [1]. In the first phase of this protocol, the source node broadcasts to the destination and the relay nodes. In the second phase, the source stops transmission, and the relay nodes forward their received signals within the first phase to the destination node. Either repetition

Manuscript received June 19, 2010; revised January 5, 2011; accepted February 3, 2011. Date of publication March 3, 2011; date of current version June 20, 2011. The work of O. Amin was supported by the Egyptian Higher Education Ministry. This paper was presented in part at the IEEE International Conference on Communication, Capetown, South Africa, May 23-27, 2010. The review of this paper was coordinated by Prof. Y. Ma.

O. Amin is with the Department of Electrical and Computer Engineering, University of British Columbia, Vancouver, BC V6T 1Z4, Canada (e-mail: oamin@ece.ubc.ca).

S. S. Ikki is with the Department of Electrical and Computer Engineering, University of Waterloo, Waterloo, ON N2L 3G1, Canada (e-mail: sikki@uwaterloo.ca).

M. Uysal is with the Faculty of Engineering, Özyegin University, Istanbul, Turkey (e-mail: murat.uysal@ ozyegin.edu.tr).

Color versions of one or more of the figures in this paper are available online at http://ieeexplore.ieee.org.

Digital Object Identifier 10.1109/TVT.2011.2121926 coding in orthogonal time slots or orthogonal space-time block coding can be used among relay nodes in the second phase. The RD protocol realizes a maximum degree of broadcasting and exhibits no receive collision. Non-orthogonal cooperation protocols such as the transmit diversity (TD) protocol and the simplified TD protocol have been further proposed [5], [6] and allow receive collision. In an effort to address the spectral inefficiency of the conventional cooperation protocols, which assume the participation of all relays, relay selection has been proposed to improve the throughput [7]. Based on a predetermined criteria, e.g., the signal-to-noise ratio (SNR), the "best" relay is selected, and only a single relay is allowed to participate in the second phase.

A common assumption in the earlier literature on cooperative diversity is the availability of perfect channel state information (CSI) at the receiver. In coherent detection, the fading channel coefficients need to first be accurately estimated during the training period, and then, these imperfect estimates are used in the detection process at the destination node. Relay nodes operating in decode-and-forward (DF) mode need the CSI of the source-to-relay channel for their own decoding process. In amplify-and-forward (AF) relaying, relay nodes might need CSI for appropriately scaling the received signal to satisfy relay power constraints. The effect of channel estimation on the overall performance of cooperative systems is therefore critical.

Several researchers have investigated the performance of cooperative systems with imperfect channel estimation [8]-[10]; however, these works are mainly limited to simulation studies. A few exceptions are [11]-[14], which aim to analytically study the impact of channel estimation for single-relay cooperative systems. In [11], Mheidat and Uysal derived a pairwise error probability (PEP) expression for the TD protocol with AF relaying. In [12], Patel and Stüber obtained an approximate error rate performance expression for the RD protocol, assuming binary phase-shift keying (PSK). In [13], Wu and Patzold derived symbol error rate expressions for the RD protocol, assuming M-ary PSK and M-ary quadrature amplitude modulation (QAM). In [14], Gedik and Uysal considered both RD and TD protocols and presented PEP analysis for mismatched-coherent and partially coherent receivers under different degrees of CSI. While the aforementioned works assume single-relay scenarios, Han et al. [15] considered multirelay AF cooperative systems and derived the average bit error rate (BER) for the RD proto$\mathrm{col}$, assuming all relays' participation in the relaying phase. 


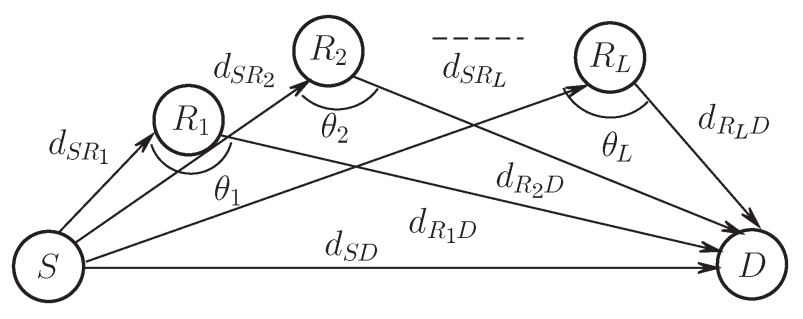

Fig. 1. Cooperative system model.

In this paper, we present a framework for the performance analysis of multirelay AF cooperative systems in the presence of channel estimation errors. We consider both conventional relaying (in which all relay nodes participate in the relaying phase) and opportunistic relaying (in which only a single relay is allowed to participate). We derive expressions for BER, outage probability, and channel capacity, demonstrating the effect of channel estimation on the performance.

The rest of this paper is organized as follows: In Section II, we present the system and channel models. In Section III, we derive the instantaneous effective SNR, which will be used for the derivations of performance measures under consideration in Section IV. The derived closed-form expressions can be used for system optimization. As an example, we use the derived BER expression to optimize power allocation in Section V. Section VI concludes this paper.

Notation: |.| denotes the absolute value. $\mathbb{E}($.$) is the expecta-$ tion operator.

\section{System And Channel Models}

As shown in Fig. 1, we consider a multirelay network in which a source node (S) and a destination node (D) communicate through a number of relay nodes denoted by $\mathrm{R}_{i}$, $i=1,2, \cdots, L$. The nodes are assumed to be located in a 2-D plane where $d_{S D}, d_{S R_{i}}$, and $d_{R_{i} D}$ denote the distances of source-to-destination $(\mathrm{S} \rightarrow \mathrm{D})$, source-to- relay $\left(\mathrm{S} \rightarrow \mathrm{R}_{i}\right)$, and relay-to-destination $\left(\mathrm{R}_{i} \rightarrow \mathrm{D}\right)$ links, respectively. The angle between lines representing $\mathrm{S} \rightarrow \mathrm{R}_{i}$ and $\mathrm{R}_{i} \rightarrow \mathrm{D}$ links is $\theta_{i}$. The complex fading coefficients between the source and the $i$ th relay and between the $i$ th relay and the destination are $h_{i}$ and $g_{i}, i=1, \ldots, L$, respectively. They are modeled as complex Gaussian with zero mean and variances of $\sigma_{h_{i}}^{2}, \sigma_{g_{i}}^{2}$, leading to Rayleigh fading. The fading coefficient for the direct link is denoted as $g_{0}$, and its amplitude distribution also follows Rayleigh distribution. Finally, in our analysis, we assume frequency-flat and time-flat (nonselective fading) channel models.

To take into account the relays' location, we also consider the long-term path loss. The path loss is inversely proportional to $d^{\alpha}$, where $d$ is the distance between nodes and $\alpha$ is the path loss exponent. By normalizing the path loss terms with respect to that of direct $\mathrm{S} \rightarrow \mathrm{D}$ link, the so-called geometrical gains [5] can be defined as $G_{S R_{i}}=\left(d_{S D} / d_{S R_{i}}\right)^{\alpha}$ and $G_{R_{i} D}=$ $\left(d_{S D} / d_{R_{i} D}\right)^{\alpha}, i=1,2 \ldots L$.

We assume the RD cooperation protocol. In the first time slot (i.e., broadcasting phase), the source broadcasts its signal. The destination and all $L$ relays receive faded noisy versions of the source signal. In the relaying phase, the source is silent.
If conventional relaying is employed, all relay nodes participate in the relaying phase and forward the scaled versions of their received signals to the destination node in orthogonal time slots. The destination node combines all signals received through indirect/direct links using maximal ratio combining. On the other hand, if opportunistic relaying is employed, the destination combines only the "best" indirect link (which yields the highest SNR at the destination) and the direct link.

Mathematically speaking, the received signals from the source at the destination and the $i$ th relay can, respectively, be written as

$$
\begin{aligned}
y_{S D} & =\sqrt{E_{S}} g_{0} x+n_{S D} \\
y_{S R_{i}} & =\sqrt{G_{S R_{i}} E_{S}} h_{i} x+n_{S R_{i}} \quad i=1,2, \ldots, L
\end{aligned}
$$

where $E_{S}$ is the source signal energy, and $x$ is either an M-PSK or an M-QAM modulated signal with unit energy. In the second time slot, the relay normalizes the received signal (to comply with power constraints) and transmits the resulting signal $x_{R_{i}}$ to the destination. The received signal at the destination from the $i$ th relay is given by

$$
y_{R_{i} D}=\sqrt{G_{R_{i} D} E_{i}} g_{i} x_{R_{i}}+n_{R_{i} D}, \quad i=1,2, \ldots, L
$$

where $E_{i}$ is the relay signal energy, and $x_{R_{i}}$ is given by

$$
x_{R_{i}}=\sqrt{\frac{1}{E_{S} G_{S R_{i}}\left|\hat{h}_{i}\right|^{2}+N_{0}}} y_{S R_{i}} .
$$

In (1)-(4), $n_{S D}, n_{S R_{i}}$, and $n_{R_{i} D}$ are zero-mean complex Gaussian noise terms with variance $N_{0} / 2$ per dimension. We assume that total power consumption in the network is given by $E_{T}$. In the case of conventional relaying, the power of source and each of $L$ relays is given by $E_{T} /(L+1)$, i.e., $E_{S}=E_{i}=$ $E_{T} /(L+1)$. On the other hand, in the case of opportunistic relaying, the source and the selected relay are each assigned $E_{T} / 2$.

The destination is assumed to have access only to imperfect channel estimates, which will be used at the combiner. Let the channel estimate of the $\mathrm{S} \rightarrow \mathrm{R}_{i}$ link be $\hat{h}_{i}$ for the $i$ th relay link. We assume that $h_{i}$ and $\hat{h}_{i}$ are jointly ergodic and stationary Gaussian processes. We can write

$$
h_{i}=\hat{h}_{i}+e_{h_{i}}
$$

where $e_{h_{i}}$ denotes the channel estimation error, which is modeled as complex Gaussian with zero mean and variance $\sigma_{e_{h_{i}}}^{2}$. Assuming a linear-minimum-mean-square-error estimator, the variance of channel estimation error is $\sigma_{e_{h_{i}}}^{2}=\mathbb{E}\left(\left|h_{i}\right|^{2}\right)$ $\mathbb{E}\left(\left|\hat{h}_{i}\right|^{2}\right)=1 /\left(N_{p} \bar{\gamma}_{S R_{i}, t}+1\right)$ [16]. Here, $N_{p}$ is the number of pilot symbols, $\bar{\gamma}_{S R_{i}, t}=\mathbb{E}\left(\gamma_{S R_{i}, t}\right)=G_{S R_{i}} E_{S, t} / N_{0}$ is the average SNR of pilot symbols for $\mathrm{S} \rightarrow \mathrm{R}_{i}$ link, and $E_{S, t}$ is the source power for training period. Similarly, variances for channel estimates of $\mathrm{S} \rightarrow \mathrm{D}$ and $\mathrm{R}_{i} \rightarrow \mathrm{D}$ links are given by $\sigma_{e_{g_{i}}}^{2}=$ $1 /\left(N_{p} \bar{\gamma}_{R_{i} D, t}+1\right)$, where $\bar{\gamma}_{S D, t}=\mathbb{E}\left(\gamma_{S D, t}\right)=E_{S, t} / N_{0}$, and 
$\bar{\gamma}_{R_{i} D, t}=\mathbb{E}\left(\gamma_{R_{i} D, t}\right)=G_{R_{i} D} E_{i, t} / N_{0}$ are the average SNRs of pilot symbols for $\mathrm{S} \rightarrow \mathrm{D}$ and $\mathrm{R}_{i} \rightarrow \mathrm{D}$ links, with $E_{i, t}$ denoting the $i$ th relay power for training period. The signal at the combiner's output can be written as [17]

$$
\lambda= \begin{cases}W_{S D} y_{S D}+\sum_{i=1}^{L} W_{i} y_{R_{i} D}, & \text { Conventional } \\ W_{S D} y_{S D}+W_{i_{\mathrm{sel}}} y_{R_{i_{\mathrm{sel}}} D}, & \text { Opportunistic }\end{cases}
$$

where the combiner coefficients are given by $W_{S D}=$ $\sqrt{E_{S}} \hat{g}_{0}^{*} / N_{0} \quad$ and $\quad W_{i}=\sqrt{G_{R_{i} D} E_{i}} \hat{h}_{i}^{*} \hat{g}_{i}^{*} /\left(\left|\hat{h}_{i}\right| N_{\text {tot }}\right)$, with $N_{\text {tot }}=N_{0}+E_{i} G_{R_{i} D}\left|\hat{g}_{i}\right|^{2} N_{0} /\left(E_{S} G_{S R_{i}}\left|\hat{h}_{i}\right|^{2}+N_{0}\right)$. In (6), $i_{\text {sel }}$ denotes the index for the selected relay, which yields the highest effective SNR in $\mathrm{S} \rightarrow \mathrm{R}_{i} \rightarrow \mathrm{D}$.

\section{Statistical Characterization of InstantaneOUs EFFECTIVE SignAL-TO-NoISE RATIO}

In this section, we will determine the probability density function (pdf) of the effective SNR (i.e., incorporating the effects of channel estimation) at the destination. Such a statistical characterization is essential for the derivation of performance measures under consideration.

\section{A. Instantaneous Effective SNR}

From (6), it can be noticed that the combiners output consist of two terms coming from either direct or indirect links. $\lambda_{S D} \stackrel{\text { def }}{=} W_{S D} y_{S D}$ is the term contributed by the $\mathrm{S} \rightarrow \mathrm{D}$ link. It can be expanded as

$$
\lambda_{S D}=\underbrace{\frac{E_{S}\left|\hat{g}_{0}\right|^{2}}{N_{0}}}_{\text {signal }} x+\underbrace{\frac{\sqrt{E_{s}} \hat{g}_{0}^{*}}{N_{0}}\left(\sqrt{E_{S}} e_{g_{0}} x+n_{S, D}\right)}_{\text {effective noise }} .
$$

The instantaneous effective SNR for this term can be therefore written as

$$
\gamma_{S D}=\frac{\hat{\gamma}_{S, D}}{E_{S} \sigma_{e_{g_{0}}}^{2} / N_{0}+1}
$$

where $\hat{\gamma}_{S D}=E_{S}\left|\hat{g}_{0}\right|^{2} / N_{0}$ is the estimated instantaneous SNR of the $\mathrm{S} \rightarrow \mathrm{D}$ link. The average effective SNR can be further written as

$$
\bar{\gamma}_{S D}=\mathbb{E}\left(\gamma_{S D}\right)=\frac{E_{S}\left(\mathbb{E}\left(\left|g_{0}\right|^{2}\right)-\sigma_{e_{g_{0}}}^{2}\right)}{\left(E_{S} \sigma_{e_{g_{0}}}^{2}+N_{0}\right)} .
$$

Similarly, it can be noticed from (5) that $\lambda_{S R_{i} D} \stackrel{\text { def }}{=} W_{i} y_{R_{i} D}$ is the term contributed by the indirect link via the $i$ th relay node. It can be expanded as in (10), shown at the bottom of the page, where $G_{i}=E_{S} G_{S R_{i}}\left|h_{i}\right|^{2}+N_{0}$. This lets us to write the corresponding instantaneous effective SNR as in (11), shown at the bottom of the page.

Further defining $\hat{\gamma}_{S R_{i}}=G_{S R_{i}} E_{S}\left|\hat{h}_{i}\right|^{2} / N_{0}$ and $\hat{\gamma}_{R_{i} D}=$ $G_{R_{i} D} E_{i}\left|\hat{g}_{i}\right|^{2} / N_{0}$ as the estimated instantaneous SNR of the $\mathrm{S} \rightarrow \mathrm{R}_{i}$ and $\mathrm{R}_{i} \rightarrow \mathrm{D}$ links, (11) can be rewritten as in (12), shown at the bottom of the page.

Equation (12) can be simplified as

$$
\gamma_{S R_{i} D}=\frac{\gamma_{S R_{i}} \gamma_{R_{i} D}}{\gamma_{S R_{i}}+\gamma_{R_{i} D}+\Omega_{i}}
$$

where $\gamma_{S R_{i}}=\hat{\gamma}_{S R_{i}} /\left(G_{S R_{i}} E_{S} \sigma_{e_{h_{i}}}^{2} / N_{0}+1\right)$ and $\gamma_{R_{i} D}=$ $\hat{\gamma}_{R_{i} D} /\left(G_{R_{i} D} E_{i} \sigma_{e_{g_{i}}}^{2} / N_{0}+1\right)$ are the instantaneous effective SNRs of $\mathrm{S} \rightarrow \mathrm{R}_{i}$ and $\mathrm{R}_{i} \rightarrow \mathrm{D}$ links, respectively, and $\Omega_{i}$ is given by

$$
\Omega_{i}=\frac{G_{S R_{i}} G_{R_{i} D} E_{S} E_{i} \sigma_{e_{h_{i}}}^{2} \sigma_{e_{g_{i}}}^{2}+G_{R_{i} D} E_{i} \sigma_{e_{g_{i}}}^{2} N_{0}+N_{0}^{2}}{\left(G_{S R_{i}} E_{S} \sigma_{e_{h_{i}}}^{2}+N_{0}\right)\left(G_{R_{i} D} E_{i} \sigma_{e_{g_{i}}}^{2}+N_{0}\right)} .
$$

$$
\lambda_{S R_{i} D}=\underbrace{\frac{\sqrt{G_{R_{i} D} E_{i}} \hat{h}_{i}^{*} \hat{g}_{e}^{*}}{\left|\hat{h}_{i}\right| N_{\mathrm{tot}}} \sqrt{\frac{G_{S R_{i}} E_{S}}{G_{i}}} h_{i} g_{i}}_{\text {signal }}+\underbrace{\sqrt{\frac{G_{R_{i} D} E_{i}}{G_{i}}} \frac{\hat{h}_{i}^{*} \hat{g}_{i}^{*}}{\left|\hat{h}_{i}\right| N_{\text {tot }}}\left(\sqrt{G_{S R_{i}} E_{S}}\left(\hat{h}_{i} e_{g_{i}}+\hat{g}_{i} e_{h_{i}}\right) x+\left(\hat{g}_{i}+e_{g_{i}}\right) n_{S, R_{i}}+\sqrt{G_{i}} n_{R_{i} D}\right)}_{\text {effective noise }}
$$

$$
\gamma_{S R_{i} D}=\frac{G_{S R_{i}} G_{R_{i} D} E_{S} E_{i}\left|\hat{h}_{i}\right|^{2}\left|\hat{g}_{i}\right|^{2}}{G_{S R_{i}} E_{S}\left|\hat{h}_{i}\right|^{2}\left(G_{R_{i} D} E_{i} \sigma_{e_{g_{i}}}^{2}+N_{0}\right)+G_{R_{i} D} E_{i}\left|\hat{g}_{i}\right|^{2}\left(G_{S R_{i}} E_{S} \sigma_{e_{h_{i}}}^{2}+N_{0}\right)+G_{S R_{i}} G_{R_{i} D} E_{S} E_{i} \sigma_{e_{h_{i}}}^{2} \sigma_{e_{g_{i}}}^{2}+G_{R_{i} D} E_{i} \sigma_{e_{g_{i}}}^{2} N_{0}+N_{0}^{2}}
$$

$$
\gamma_{S R_{i} D}=\frac{\hat{\gamma}_{S R_{i}} \hat{\gamma}_{R_{i} D}}{\hat{\gamma}_{S R_{i}}\left(\frac{G_{R_{i} D} E_{i} \sigma_{e_{g_{i}}}^{2}}{N_{0}}+1\right)+\hat{\gamma}_{R_{i} D}\left(\frac{G_{S R_{i}} E_{S} \sigma_{e_{h_{i}}}^{2}}{N_{0}}+1\right)+\frac{G_{S R_{i}} G_{R_{i} D} E_{S} E_{i} \sigma_{e_{h_{i}}}^{2} \sigma_{e_{g_{i}}}^{2}}{N_{0}^{2}}+\frac{G_{R_{i} D} E_{i} \sigma_{e_{g_{i}}}^{2}}{N_{0}}+1}
$$


Finally, using (8) and (13), the effective total output SNR can be obtained as

$$
\gamma_{\text {tot }}= \begin{cases}\gamma_{S D}+\sum_{i=1}^{L} \gamma_{S R_{i} D}, & \text { Conventional } \\ \gamma_{S D}+\max _{i \in L} \gamma_{S R_{i} D}, & \text { Opportunistic. }\end{cases}
$$

To simplify the ensuing performance analysis, we employ a tight upper bound on $\gamma_{S R_{i} D}$, which is given as $\gamma_{S R_{i} D} \leq \gamma_{i}=$ $\min \left(\gamma_{S R_{i}}, \gamma_{R_{i} D}\right)$ [17]. This yields bounds on (15) as

$$
\gamma_{u b}^{\text {Con }}=\gamma_{S D}+\sum_{i=1}^{L} \min \left(\gamma_{S R_{i}}, \gamma_{R_{i} D}\right)
$$

for conventional relaying. Similarly, we have

$$
\gamma_{u b}^{\mathrm{Opp}}=\gamma_{S D}+\max _{i \in L} \min \left(\gamma_{S R_{i}}, \gamma_{R_{i} D}\right)
$$

for opportunistic relaying.

\section{B. PDFs of the Instantaneous Effective SNR}

For conventional relaying, the pdf of (16) can be derived, following the steps in [18], which yields the following:

$$
f_{\gamma_{u b}^{\text {Con }}}(\gamma)=\frac{\beta_{S, D}}{\bar{\gamma}_{S D}} \exp \left(\frac{-\gamma}{\bar{\gamma}_{S D}}\right)+\sum_{i=1}^{L} \frac{\beta_{i}}{\bar{\gamma}_{i}} \exp \left(\frac{-\gamma}{\bar{\gamma}_{i}}\right)
$$

where

$$
\begin{aligned}
\bar{\gamma}_{i} & =\frac{\bar{\gamma}_{S R_{i}} \bar{\gamma}_{R_{i} D}}{\bar{\gamma}_{S R_{i}}+\bar{\gamma}_{R_{i} D}} \\
\bar{\gamma}_{S R_{i}} & =\mathbb{E}\left(\gamma_{S R_{i}}\right)=\frac{G_{S R_{i}} E_{S}\left(\mathbb{E}\left(\left|h_{i}\right|^{2}\right)-\sigma_{e_{h_{i}}}^{2}\right)}{\left(G_{S R_{i}} E_{S} \sigma_{e_{h_{i}}}^{2}+N_{0}\right)} \\
\bar{\gamma}_{R_{i} D} & =\mathbb{E}\left(\gamma_{R_{i} D}\right)=\frac{G_{R_{i} D} E_{S}\left(\mathbb{E}\left(\left|g_{i}\right|^{2}\right)-\sigma_{e_{g_{i}}}^{2}\right)}{\left(G_{R_{i} D} E_{S} \sigma_{e_{g_{i}}}^{2}+N_{0}\right)} \\
\beta_{S D} & =\prod_{i=1}^{L}\left(1-\frac{\bar{\gamma}_{i}}{\bar{\gamma}_{S D}}\right)^{-1} \\
\beta_{i} & =\left(1-\frac{\bar{\gamma}_{S D}}{\bar{\gamma}_{i}}\right)^{-1} \prod_{k=1, k \neq i}^{L}\left(1-\frac{\bar{\gamma}_{k}}{\bar{\gamma}_{i}}\right)^{-1} .
\end{aligned}
$$

For opportunistic relaying, the pdf of (17) is obtained as

$$
\begin{aligned}
& f_{\gamma_{u b}^{\mathrm{Opp}}}(\gamma)=\sum_{i=1}^{L}(-1)^{i+1} \sum_{K_{1}=1}^{L-i+1} \sum_{K_{2}=K_{1}+1}^{L-i+2} \cdots \\
& \sum_{K_{i}=K_{i-1}+1}^{L} \frac{1}{1 / \xi_{i}-\bar{\gamma}_{S D}}\left(\exp (-\gamma \xi)-\exp \left(-\frac{\gamma}{\bar{\gamma}_{S D}}\right)\right)
\end{aligned}
$$

where $\xi_{i}=\sum_{j=1}^{i}\left(1 / \bar{\gamma}_{K_{j}}\right)$. Considering independent identical distribution (i.i.d) channels, i.e., $\bar{\gamma}_{S D}=\bar{\gamma}_{S R_{i}}=\bar{\gamma}_{R_{i} D}=\bar{\gamma}$,
(24) reduces to

$$
\begin{aligned}
f_{\gamma_{u b, i i d}}^{\text {Opp }}(\gamma)=\sum_{q=1}^{M}\left(\begin{array}{c}
M \\
q
\end{array}\right) \frac{(-1)^{q-1}}{\bar{\gamma}(1-1 /(2 q))} \\
\times\left[\exp \left(-\frac{\gamma}{\bar{\gamma}}\right)-\exp \left(-\frac{2 q \gamma}{\bar{\gamma}}\right)\right] .
\end{aligned}
$$

\section{Performance Analysis}

\section{A. Error Probability}

The average error probability over frequency-flat fading channels can be found by averaging the conditional error probability in additive white Gaussian noise $P_{b}\left(e / \gamma_{u b}\right)$. Mathematically, $P_{b}(e)$ is given by [19]

$$
P_{b}(e)=\int_{0}^{\infty} P_{b}\left(e / \gamma_{u b}\right) f_{\gamma_{u b}}\left(\gamma_{u b}\right) d \gamma_{u b}
$$

where $f_{\gamma_{u b}}\left(\gamma_{u b}\right)$ is given by either (18) and (24) for the schemes under consideration. Note that, for several Gray bit-mapped constellations, $P_{b}\left(e / \gamma_{u b}\right)$ is in the form of $a \operatorname{erfc}\left(\sqrt{b \gamma_{u b}}\right)$, with $\operatorname{erfc}(x)$ being the complementing error function [19], and $a$, $b$ are constants, depending on the type of modulation (e.g., BPSK: $a=0.5$, and $b=1$, QPSK: $a=0.5$, and $b=0.5$ ). By substituting (18) into (26) and solving the integration, a lower bound on the error probability for conventional relaying can be obtained as

$$
P_{b}^{\text {Con }}(e)=a \beta_{S D}\left(1-\sqrt{\frac{b \bar{\gamma}_{S D}}{1+b \bar{\gamma}_{S D}}}\right)+a \sum_{i=1}^{L} \beta_{i}\left(1-\sqrt{\frac{b \bar{\gamma}_{i}}{1+b \bar{\gamma}_{i}}}\right)
$$

Similarly, by substituting (24) into (26) and performing the integration operation, we find a lower bound on the error probability for opportunistic relaying as

$$
\begin{gathered}
P_{b}^{\mathrm{Opp}}(e)=a \sum_{i=1}^{L}(-1)^{i+1} \sum_{K_{1}=1}^{L-i+1} \sum_{K_{2}=K_{1}+1}^{L-i+2} \cdots \sum_{K_{i}=K_{i-1}+1}^{L} \\
\times 1-\frac{1}{1-\bar{\gamma}_{S D} \xi_{i}} \sqrt{\frac{b}{\xi_{i}+b}}+\frac{\bar{\gamma}_{S D} \xi_{i}}{1-\bar{\gamma}_{S D} \xi_{i}} \sqrt{\frac{b \bar{\gamma}_{S D}}{1+b \bar{\gamma}_{S D}}} .
\end{gathered}
$$

As a sanity check, it can be noted that, in the perfect CSI case (i.e., $\sigma_{e_{h_{i}}}^{2}=\sigma_{e_{g_{i}}}^{2}=0$ ), (28) reduces to the expression given by [20, eq. (14)].

To provide some further insight into the performance, we now consider the asymptotically high-SNR case. Following [21], we can obtain an approximation to (27) as follows:

$$
P_{b}^{\mathrm{Con}}(e) \approx \frac{a C(L)}{b^{L+1}} \frac{1}{\bar{\gamma}_{S D}} \prod_{i=1}^{L}\left(\frac{1}{\bar{\gamma}_{S R_{i}}}+\frac{1}{\bar{\gamma}_{R_{i} D}}\right)
$$

where $C(L)=\prod_{i=1}^{L+1}(2 i-1) /(2(L+1) !)$ is constant, which depends on the number of relay nodes $L$. It is observed from (29) that a full diversity order of $L+1$ is achieved as in the case of the perfect channel estimation. For $\sigma_{e_{h_{i}}}^{2}=\sigma_{e_{g_{i}}}^{2}=0$, 
(29) reduces to [22, eq. (21)], which is reported for perfect CSI case.

An asymptotical expression can be obtained for opportunistic relaying as (see the Appendix for the proof)

$$
P_{b}^{\mathrm{Opp}}(e) \approx \frac{a U(L)}{b^{L+1}} \frac{1}{\bar{\gamma}_{S D}} \prod_{i=1}^{L}\left(\frac{1}{\bar{\gamma}_{S R_{i}}}+\frac{1}{\bar{\gamma}_{R_{i} D}}\right)
$$

where $U(L)=L \prod_{i=1}^{L+1}(2 i-1) /(2(L+1)$ !). A comparison of (29) and (30) reveals that both expressions have identical forms where the only difference comes from the coefficient term given by $U(L)=L C(L)$. This will eventually result in a horizontal shift between the performance of two schemes, both of which are able to achieve the full diversity.

\section{B. Outage Probability}

The mutual information between the source and the destination for conventional and opportunistic relaying can be written as

$$
\begin{aligned}
& I_{\text {Con }}=\frac{1}{L+1} \log _{2}\left(1+\gamma_{u b}^{\mathrm{Con}}\right) \\
& I_{\mathrm{Opp}}=\frac{1}{2} \log _{2}\left(1+\gamma_{u b}^{\mathrm{Opp}}\right) .
\end{aligned}
$$

The reason for different factors in (31) and (32) is that we need $L+1$ time slots (or orthogonal channels) to transmit the data in conventional relaying and only two times slots in the case of opportunistic relaying.

The outage probability is defined as the probability that the mutual information falls below the required rate $r$. For conventional relaying, it is given by

$P_{\text {out }}^{\text {Con }}=\operatorname{Pr}\left(I_{\text {Reg }} \leq r\right)=\operatorname{Pr}\left(\gamma_{u b}^{\text {Con }} \leq \gamma_{t}^{\text {Con }}=2^{(L+1) r}-1\right)$.

It can be noted that outage probability is actually the cumulative CDF of $\gamma_{\text {out }}^{\text {Conv }}$ evaluated at $2^{(L+1) r}-1$. Using (18), we can obtain a lower bound on the outage probability as

$$
\begin{aligned}
P_{\text {out }}^{\text {Con }}=\beta_{S D}(1-\exp & \left.\left(-\frac{\gamma_{t}^{\text {Con }}}{\bar{\gamma}_{S D}}\right)\right) \\
& +\sum_{i=1}^{L} \beta_{i}\left(1-\exp \left(-\frac{\gamma_{t}^{\text {Con }}}{\bar{\gamma}_{i}}\right)\right)
\end{aligned}
$$

where $\beta_{S D}$ and $\beta_{i}$ are earlier defined in (22) and (23). Asymptotically, it can be approximated as

$$
P_{\mathrm{out}}^{\mathrm{Con}}(e) \approx \frac{\left[2^{(L+1) r}-1\right]^{L+1}}{\bar{\gamma}_{S D}(L+1) !} \prod_{i=1}^{L}\left(\frac{1}{\bar{\gamma}_{S R_{i}}}+\frac{1}{\bar{\gamma}_{R_{i} D}}\right) .
$$

Similarly, for opportunistic relaying, we obtain

$$
\begin{aligned}
P_{\text {out }}^{\text {Opp }} & =\operatorname{Pr}\left(I_{\mathrm{Opp}} \leq r\right)=\operatorname{Pr}\left(\gamma_{u b}^{\text {Opp }} \leq \gamma_{t}^{\text {Opp }}=2^{2 r}-1\right) \\
& =\sum_{i=1}^{L}(-1)^{i+1} \sum_{K_{1}=1}^{L-i+1} \sum_{K_{2}=K_{1}+1}^{L-i+2} \ldots \sum_{K_{i}=K_{i-1}+1}^{L} 1
\end{aligned}
$$

$$
\begin{gathered}
-\frac{1}{1-\bar{\gamma}_{S D} \xi_{i}} \exp \left(-\gamma_{t}^{\mathrm{Opp}} \xi_{i}\right) \\
+\frac{\bar{\gamma}_{S, D} \xi_{i}}{1-\bar{\gamma}_{S D} \xi_{i}} \exp \left(-\frac{\gamma_{t}^{\mathrm{Opp}}}{\bar{\gamma}_{S D}}\right) .
\end{gathered}
$$

Asymptotically, this yields

$$
P_{\mathrm{out}}^{\mathrm{Opp}}(e) \approx \frac{\left[2^{2 r}-1\right]^{L+1}}{(L+1)} \frac{1}{\bar{\gamma}_{S D}} \prod_{i=1}^{L}\left(\frac{1}{\bar{\gamma}_{S R_{i}}}+\frac{1}{\bar{\gamma}_{R_{i} D}}\right) .
$$

\section{Ergodic Channel Capacity}

For conventional relaying, the average channel capacity is given by

$$
\bar{C}^{\text {Con }}=\frac{W}{L+1} \int_{0}^{\infty} \log _{2}(1+\gamma) f_{\gamma_{u b}^{\text {Con }}}(\gamma) d \gamma .
$$

By substituting (18) into (38), an upper bound on channel capacity can be obtained in a closed form as

$$
\begin{aligned}
\bar{C}^{\text {Con }}=\frac{W}{(L+1) \ln (2)}[ & \beta_{S D} \exp \left(\bar{\gamma}_{S D}^{-1}\right) \mathrm{E}_{1}\left(\bar{\gamma}_{S D}^{-1}\right) \\
& \left.+\sum_{i=1}^{L} \beta_{i} \exp \left(\bar{\gamma}_{i}^{-1}\right) \mathrm{E}_{1}\left(\bar{\gamma}_{i}^{-1}\right)\right] .
\end{aligned}
$$

where $\mathrm{E}_{1}(x)$ is the exponential integral defined as $\mathrm{E}_{1}(x)=$ $\int_{x}^{\infty} \exp (-t) / t d t$ [23]. It can be readily checked that, for $\sigma_{e_{h_{i}}}^{2}=$ $\sigma_{e_{g_{i}}}^{2}=0$, (39) reduces to [24, eq. (32)] reported for perfect CSI scenario.

For opportunistic relaying, we have

$$
\bar{C}^{\mathrm{Opp}}=\frac{W}{2} \int_{0}^{\infty} \log _{2}(1+\gamma) f_{\gamma_{u b}^{\mathrm{Opp}}}(\gamma) d \gamma .
$$

For this case, we obtain an upper bound as

$$
\begin{aligned}
\bar{C}^{\text {Opp }} & =\frac{W}{2 \ln (2)} \sum_{i=1}^{L}(-1)^{i+1} \sum_{K_{1}=1}^{L-i+1} \cdots \sum_{K_{i}=K_{i-1}+1}^{L} \\
\times & \frac{\exp \left(\xi_{i}\right) E_{1}\left(\xi_{i}\right)-\bar{\gamma}_{S D} \xi_{i} \exp \left(1 / \bar{\gamma}_{S D}\right) E_{1}\left(1 / \bar{\gamma}_{S D}\right)}{1-\bar{\gamma}_{S D} \xi_{i}} .
\end{aligned}
$$

For $\sigma_{e_{h_{i}}}^{2}=\sigma_{e_{g_{i}}}^{2}=0,(41)$ reduces to [25, eq. (24)] reported for perfect CSI scenario.

\section{Adaptive Power Allocation (APA)}

The derived closed-form expressions can be used for system optimization. In this section, as an example, we consider the derived error probability expression and obtain the optimal power distribution rules between the source and the relay nodes to minimize it. 


\section{A. Conventional Relaying}

For conventional relaying, the optimization problem can be formulated as

$$
\min _{\text {s.t. } E_{S}+\sum_{i=1}^{L} E_{i}=E_{T}} P^{\mathrm{Con}}(e) .
$$

The power to be allocated to source and relay nodes can be written in terms of total power as $E_{S}=\rho_{0} E_{T}, E_{i}=\rho_{i} E_{T}, i=$ $1,2, \ldots, L$. Using (29), (42) can be rewritten as

$$
\min _{\text {s.t. } E_{s}+\sum_{i=1}^{L} E_{i}=E_{T}}\left(\epsilon_{0}+\frac{\alpha_{0}}{\rho_{0} E_{T}}\right) \prod_{i=1}^{L}\left(\epsilon_{i}+\frac{\alpha_{i}}{\rho_{0} E_{T}}+\frac{\beta_{i}}{\rho_{i} E_{T}}\right)
$$

where $\epsilon_{0}, \epsilon_{i}, \alpha_{0}$, and $\alpha_{i}$ are given by

$$
\begin{aligned}
\epsilon_{0} & =\frac{\sigma_{e_{g_{0}}} a C(L)}{b^{L+1}\left(\mathbb{E}\left|g_{0}\right|^{2}-\sigma_{e_{g_{0}}}^{2}\right)} \\
\epsilon_{i} & =\frac{\sigma_{e_{h_{i}}}^{2}}{\mathbb{E}\left(\left|h_{i}\right|^{2}\right)-\sigma_{e_{h_{i}}}^{2}}+\frac{\sigma_{e_{g_{i}}}^{2}}{\mathbb{E}\left(\left|g_{i}\right|^{2}\right)-\sigma_{e_{g_{i}}}^{2}} \\
\alpha_{0} & =\frac{a N_{0} C(L)}{b^{L+1}\left(\mathbb{E}\left(\left|g_{0}\right|^{2}\right)-\sigma_{e_{g_{0}}}^{2}\right)} \\
\alpha_{i} & =\frac{N_{0}}{G_{S R_{i}}\left(\mathbb{E}\left(\left|h_{i}\right|^{2}\right)-\sigma_{e_{h_{i}}}^{2}\right)} \\
\beta_{i} & =\frac{N_{0}}{G_{R_{i} D}\left(\mathbb{E}\left(\left|g_{i}\right|^{2}\right)-\sigma_{e_{g_{i}}}^{2}\right)} .
\end{aligned}
$$

Unfortunately, (43) cannot be solved in a closed form; however, $\rho_{i}, i=1,2, \ldots, L$ can be calculated through numerical optimization techniques. For perfect channel estimation (i.e., $\left.\sigma_{e_{h_{i}}}^{2}=\sigma_{e_{g_{i}}}^{2}=0, i=0, \ldots, L\right),(43)$, however, simplifies to

$$
\min _{\text {s.t. } E_{s}+\sum_{i=1}^{L} E_{i}=E_{T}} \frac{a C(L) N_{0}^{L+1}}{b^{L+1} \rho_{0} E_{T}^{L+1}} \prod_{i=1}^{L}\left(\frac{1}{G_{S R_{i}} \rho_{0}}+\frac{1}{G_{R_{i} D} \rho_{i}}\right) .
$$

Using Lagrange multiplier method, we can obtain optimum values for $\rho_{i}, i=1,2, \ldots, L$ as

$$
\rho_{i}=\frac{-G_{S R_{i}} \rho_{0}+\sqrt{G_{S R_{i}}^{2} \rho_{0}^{2}+4 G_{R_{i} D} G_{S R_{i}} \rho_{0} /(L+1)}}{2 G_{R_{i} D}}
$$

where $\rho_{0}$ can be computed by solving

$$
\begin{aligned}
\sum_{i=1}^{L} \sqrt{\left(\frac{G_{S R_{i}}}{G_{R_{i} D}}\right)^{2} \rho_{0}^{2}}+\frac{4}{(L+1)}\left(\frac{G_{S R_{i}}}{G_{R_{i} D}}\right) \rho_{0} \\
-2\left(1-\rho_{0}\right)-\rho_{0} \sum_{i=1}^{L} \frac{G_{S R_{i}}}{G_{R_{i} D}}=0
\end{aligned}
$$

using any minimization bracketing method [26].
In Table I, we present the optimal values of $\rho_{i}, i=$ $0,1, \ldots, L$ for both perfect and imperfect channel estimation cases. We assume 4-PSK modulation, $\alpha=2$, and $N_{p}=1$ and consider the following scenarios based on the number of relays and relay geometry.

1) $L=1, \theta_{1}=\pi$, and $G_{S R_{1}} / G_{R_{1} D}=-30 \mathrm{~dB}$.

2) $L=2, \theta_{1}=\pi, \theta_{2}=\pi / 3, G_{S R_{1}} / G_{R_{1} D}=-30 \mathrm{~dB}$, and $G_{S R_{2}} / G_{R_{2} D}=0 \mathrm{~dB}$.

3) $L=2, \theta_{1}=\pi, \theta_{2}=\pi / 3, \theta_{3}=\pi / 6, G_{S R_{1}} / G_{R_{1} D}=$ $-30 \mathrm{~dB}, G_{S R_{2}} / G_{R_{2} D}=0 \mathrm{~dB}$, and $G_{S R_{3}} / G_{R_{3} D}=$ $30 \mathrm{~dB}$.

4) i.i.d case: $G_{S D}=G_{S R_{i}}=G_{R_{i} D}=1$, and $\theta_{i}=\pi / 3$, $i=1,2,3$.

\section{B. Opportunistic Relaying}

For opportunistic relaying, the optimization problem can be formulated as

$$
\min _{\text {s.t. } E_{s}+E_{R_{\mathrm{sel}}}=E_{T}} P^{\mathrm{Opp}}(e) \text {. }
$$

The power to be allocated to source and selected relay can be written in terms of the total power as $E_{S}=\rho E_{T}$ and $E_{i}=$ $(1-\rho) E_{T}$. Using (30), (52) can be rewritten as

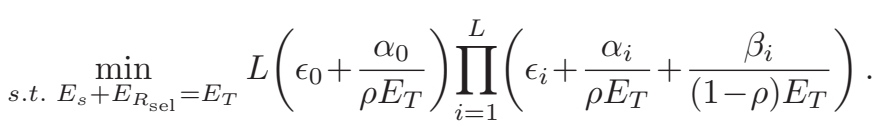

The optimal $\rho$ parameter value can be calculated from equating $\partial P^{\mathrm{Opp}}(e) / \partial \rho$ to zero, which yields

$$
\left(\epsilon_{0}+\frac{\alpha_{0}}{\rho E_{T}}\right) \sum_{k=1}^{L} \frac{\frac{\beta_{k}}{(1-\rho)^{2} E_{T}}-\frac{\alpha_{k}}{\rho^{2} E_{T}}}{\epsilon_{k}+\frac{\alpha_{k}}{\rho E_{T}}+\frac{\beta_{k}}{(1-\rho) E_{T}}}-\frac{\alpha_{0}}{\rho^{2} E_{T}}=0 .
$$

In the case of perfect channel estimation, (54) simplifies to

$$
(L+1) \rho-1-\sum_{k=1}^{L} \frac{(1-\rho) G_{R_{i} D} \mathbb{E}\left(\left|g_{i}\right|^{2}\right)}{\rho G_{S R_{i}} \mathbb{E}\left(\left|h_{i}\right|^{2}\right)+(1-\rho) G_{R_{i} D} \mathbb{E}\left(\left|g_{i}\right|^{2}\right)}=0 .
$$

Further imposing the assumption of identical channels (i.e., $\mathbb{E}\left(\left|g_{i}\right|^{2}\right)=\mathbb{E}\left(\left|h_{i}\right|^{2}\right)$, and $\left.G_{S D}=G_{S R_{i}}=G_{R_{i} D}=1\right)$, optimal $\rho$ is found as

$$
\rho_{\text {iden }}=\frac{L+1}{2 L+1} .
$$

Optimal values of $\rho$ for aforementioned scenarios with perfect and imperfect channel estimation can be found in Table II. From Tables I and II, we observe that power allocation values slightly vary with SNR for the case of imperfect channel estimation (as a result of the dependency of channel estimation error on SNR). On the other hand, they are independent of SNR in the case of perfect channel estimation.

\section{Simulation Results AND Discussion}

In this section, we provide numerical results to confirm the derived analytical expressions. We assume the same system 
TABLE I

Optimal Power Allocation Values for Conventional Relaying. (a) Imperfect Channel Estimation. (b) Imperfect Channel Estimation and i.i.d CAse. (c) Perfect Channel Estimation

(a)

\begin{tabular}{|c|c|c|c|}
\hline $\begin{array}{c}\mathbf{S N R} \\
(\mathbf{d B})\end{array}$ & $\begin{array}{c}\boldsymbol{L}=\mathbf{1} \\
\rho_{0}, \rho_{1}\end{array}$ & $\begin{array}{c}\boldsymbol{L}=\mathbf{2} \\
\rho_{0}, \rho_{1}, \rho_{2}\end{array}$ & $\begin{array}{c}\boldsymbol{L}=\mathbf{3} \\
\rho_{0}, \rho_{1}, \rho_{2}, \rho_{3}\end{array}$ \\
\hline $\mathbf{0}$ & $0.9871-0.0129$ & $0.7209-0.0076-0.2715$ & $0.5564-0.0625-0.0625-0.3186$ \\
\hline $\mathbf{4}$ & $0.9835-0.0165$ & $0.7107-0.0100-0.2793$ & $0.5576-0.0625-0.0625-0.3174$ \\
\hline $\mathbf{8}$ & $0.9809-0.0191$ & $0.7040-0.0119-0.2841$ & $0.6250-0.1250-0.1250-0.1250$ \\
\hline $\mathbf{1 2}$ & $0.9794-0.0206$ & $0.7006-0.0131-0.2863$ & $0.5002-0.0091-0.2031-0.2876$ \\
\hline $\mathbf{1 6}$ & $0.9787-0.0213$ & $0.6991-0.0137-0.2872$ & $0.4983-0.0097-0.2038-0.2882$ \\
\hline $\mathbf{2 0}$ & $0.9784-0.0216$ & $0.6984-0.0140-0.2876$ & $0.4975-0.0099-0.2041-0.2884$ \\
\hline $\mathbf{2 4}$ & $0.9783-0.0217$ & $0.6982-0.0141-0.2877$ & $0.4972-0.0100-0.2042-0.2885$ \\
\hline $\mathbf{2 8}$ & $0.9782-0.0218$ & $0.6981-0.0141-0.2878$ & $0.4971-0.0101-0.2043-0.2886$ \\
\hline
\end{tabular}

(b)

\begin{tabular}{|c|c|c|c|}
\hline $\begin{array}{c}\text { SNR } \\
\text { (dB) }\end{array}$ & $\begin{array}{c}\boldsymbol{L}=\mathbf{1} \text { (i.i.d.) } \\
\rho_{0}, \rho_{1}\end{array}$ & $\begin{array}{c}\boldsymbol{L}=\mathbf{2} \text { (i.i.d.) } \\
\rho_{0}, \rho_{1}, \rho_{2}\end{array}$ & $\begin{array}{c}\boldsymbol{L}=\mathbf{3} \text { (i.i.d.) } \\
\rho_{0}, \rho_{1}, \rho_{2}, \rho_{3}\end{array}$ \\
\hline $\mathbf{0}$ & $0.6558-0.3442$ & $0.5245-0.2378-0.2378$ & $0.4511-0.1830-0.1830-0.1830$ \\
\hline $\mathbf{4}$ & $0.6518-0.3482$ & $0.5196-0.2402-0.2402$ & $0.4635-0.1793-0.1793-0.1780$ \\
\hline $\mathbf{8}$ & $0.6493-0.3507$ & $0.5162-0.2419-0.2419$ & $0.6250-0.1250-0.1250-0.1250$ \\
\hline $\mathbf{1 2}$ & $0.6481-0.3519$ & $0.5143-0.2428-0.2428$ & $0.4402-0.1866-0.1866-0.1866$ \\
\hline $\mathbf{1 6}$ & $0.6475-0.3525$ & $0.5135-0.2432-0.2432$ & $0.4392-0.1869-0.1869-0.1869$ \\
\hline $\mathbf{2 0}$ & $0.6473-0.3527$ & $0.5132-0.2434-0.2434$ & $0.4388-0.1871-0.1871-0.1871$ \\
\hline $\mathbf{2 4}$ & $0.6472-0.3528$ & $0.5130-0.2435-0.2435$ & $0.4386-0.1871-0.1871-0.1871$ \\
\hline
\end{tabular}

(c)

\begin{tabular}{|c|c|c|c|c|c|}
\hline $\boldsymbol{L}=\mathbf{1}$ & $\boldsymbol{L}=\mathbf{1}$ (i.i.d.) & $\boldsymbol{L}=\mathbf{2}$ & $\boldsymbol{L}=\mathbf{2}$ (i.i.d.) & $\boldsymbol{L}=\mathbf{3}$ & $\boldsymbol{L}=\mathbf{3}$ (i.i.d.) \\
\hline 0.9784 & 0.6667 & 0.7357 & 0.5352 & 0.5519 & 0.4606 \\
\hline 0.0216 & 0.3333 & 0.0153 & 0.2324 & 0.0115 & 0.1798 \\
\hline & & 0.2490 & 0.2324 & 0.1868 & 0.1798 \\
\hline & & & & 0.2499 & 0.1798 \\
\hline
\end{tabular}

TABLE II

Optimal $\rho$ Values for Opportunistic Relaying $(L=1,2,3)$. (a) Imperfect Channel Estimation. (b) Perfect Channel Estimation

(a)

\begin{tabular}{|c|c|c|c|}
\hline $\begin{array}{c}\mathbf{S N R} \\
\mathbf{d B}\end{array}$ & $\begin{array}{c}\boldsymbol{L}=\mathbf{1} \\
\rho_{0}, \rho_{1}\end{array}$ & $\begin{array}{c}\boldsymbol{L}=\mathbf{2} \\
\rho_{0}, \rho_{1}, \rho_{2}\end{array}$ & $\begin{array}{c}\boldsymbol{L}=\mathbf{3} \\
\rho_{0}, \rho_{1}, \rho_{2}, \rho_{3}\end{array}$ \\
\hline $\mathbf{0}$ & $0.9871-0.0129$ & $0.7209-0.0076-0.2715$ & $0.5564-0.0625-0.0625-0.3186$ \\
\hline $\mathbf{4}$ & $0.9835-0.0165$ & $0.7107-0.0100-0.2793$ & $0.5576-0.0625-0.0625-0.3174$ \\
\hline $\mathbf{8}$ & $0.9809-0.0191$ & $0.7040-0.0119-0.2841$ & $0.6250-0.1250-0.1250-0.1250$ \\
\hline $\mathbf{1 2}$ & $0.9794-0.0206$ & $0.7006-0.0131-0.2863$ & $0.5002-0.0091-0.2031-0.2876$ \\
\hline $\mathbf{1 6}$ & $0.9787-0.0213$ & $0.6991-0.0137-0.2872$ & $0.4983-0.0097-0.2038-0.2882$ \\
\hline $\mathbf{2 0}$ & $0.9784-0.0216$ & $0.6984-0.0140-0.2876$ & $0.4975-0.0099-0.2041-0.2884$ \\
\hline $\mathbf{2 4}$ & $0.9783-0.0217$ & $0.6982-0.0141-0.2877$ & $0.4972-0.0100-0.2042-0.2885$ \\
\hline $\mathbf{2 8}$ & $0.9782-0.0218$ & $0.6981-0.0141-0.2878$ & $0.4971-0.0101-0.2043-0.2886$ \\
\hline
\end{tabular}

(b)

\begin{tabular}{|c|c|c|c|c|c|}
\hline $\boldsymbol{L}=\mathbf{1}$ & $\boldsymbol{L}=\mathbf{1}$ (i.i.d.) & $\boldsymbol{L}=\mathbf{2}$ & $\boldsymbol{L}=\mathbf{2}$ (i.i.d.) & $\boldsymbol{L}=\mathbf{3}$ & $\boldsymbol{L}=\mathbf{3}$ (i.i.d.) \\
\hline 0.9784 & 0.6667 & 0.7493 & 0.6000 & 0.5998 & 0.5714 \\
\hline
\end{tabular}

parameters for non-identical channels considered in Tables I and II.

In Fig. 2, we present the error probability performance of conventional relaying for $L=1,2$ and 3 . In this figure, the exact error probability (obtained through simulations) is plotted along with the lower bound given by (27) and the asymptotical expression by (29). The derived lower bound lies within $0.3 \mathrm{~dB}$ of the exact performance. The asymptotical expression further provides an excellent match in moderate-high SNR region ( $>15 \mathrm{~dB})$. The performance with perfect CSI is also included as a benchmark. It is observed that, due to imperfect channel estimation, the performance is degraded by $\sim 3 \mathrm{~dB}$. However, the slope of the performance curves and, therefore, the diversity order remain the same for both perfect and imperfect CSI.
In Fig. 3, we present the error probability performance of opportunistic relaying for $L=1,2$ and 3 . In this figure, the lower bound given by (28) and the asymptotical expression given by (30) are provided along with the exact (simulated) expression. Similar to Fig. 2, the lower bound is within $0.3 \mathrm{~dB}$ of the exact performance, and the asymptotical expression provides tight results for SNR higher than $15 \mathrm{~dB}$. We further observe that the performance is degraded by 3, 4, and $4.7 \mathrm{~dB}$ as a result of the imperfect channel estimation.

From the comparison of Figs. 2 and 3, we observe that opportunistic relaying outperforms conventional relaying, confirming the earlier reported results for the case of perfect channel estimation (see, e.g., [27]-[30]). It is also important to emphasize that imperfect channel estimates affect the opportunistic relaying more than the conventional relaying. This is due to the 


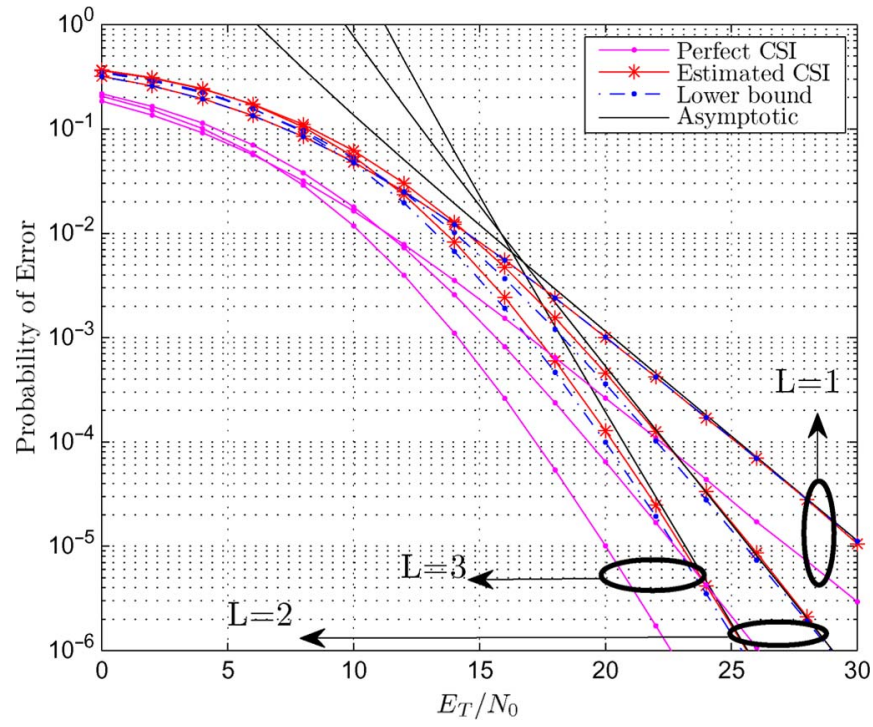

Fig. 2. Probability of error for conventional relaying.

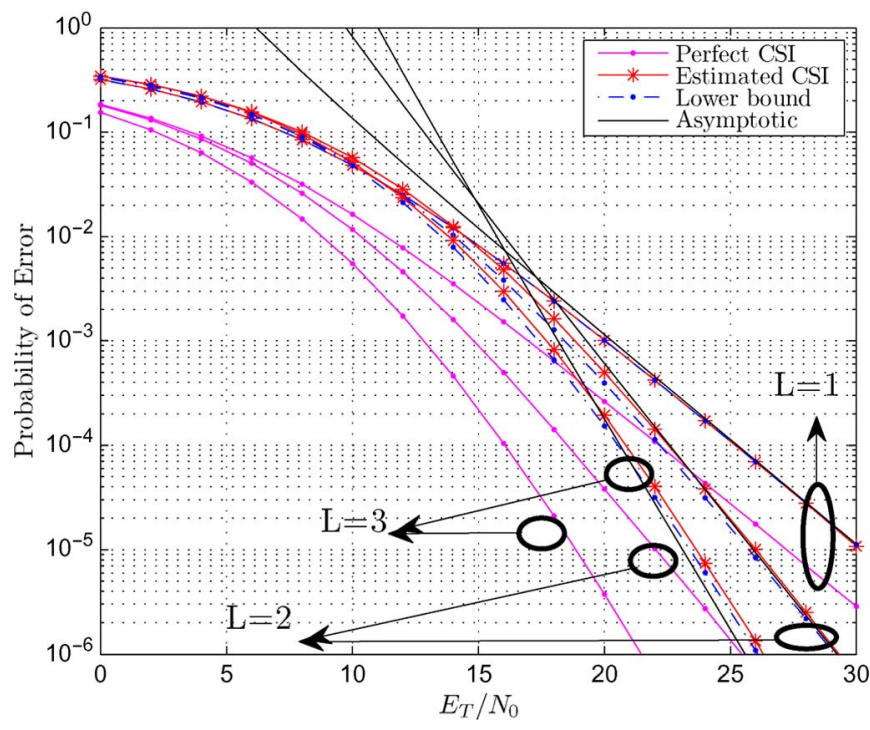

Fig. 3. Probability of error for opportunistic relaying.

effect of imperfect channel estimation on both relay selection and the MRC combining processes in opportunistic relaying. On the other hand, it has impact only on the MRC combiner in the case of conventional relaying.

In Fig. 4, we return our attention to outage probability. Lower bounds on the outage probability for conventional and opportunistic relaying, respectively, given by (34) and (36), along with asymptotical expressions given by (35) and (37), are illustrated, assuming two relays. As expected from the similarity of corresponding expressions, tightness is similar to that observed in error probability.

In Fig. 5, we illustrate the upper bounds on ergodic channel capacity for conventional and opportunistic relaying, assuming $L=2$ and 3 . The degradation in the capacity for conventional relaying due to imperfect channel estimation is $3 \mathrm{~dB}$ for both $L=2$ and 3 . The degradation in opportunistic relaying climbs to 4 and $4.9 \mathrm{~dB}$ for $L=2$ and 3 , respectively. Despite the additional degradation, opportunistic relaying outperforms the

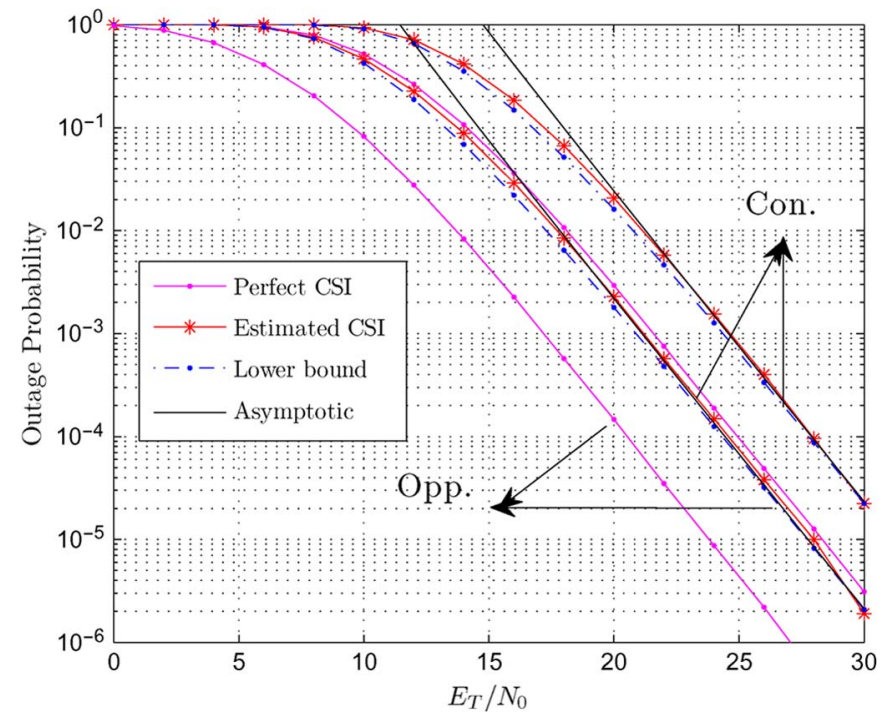

Fig. 4. Outage probability for conventional and opportunistic relaying with $L=2$.

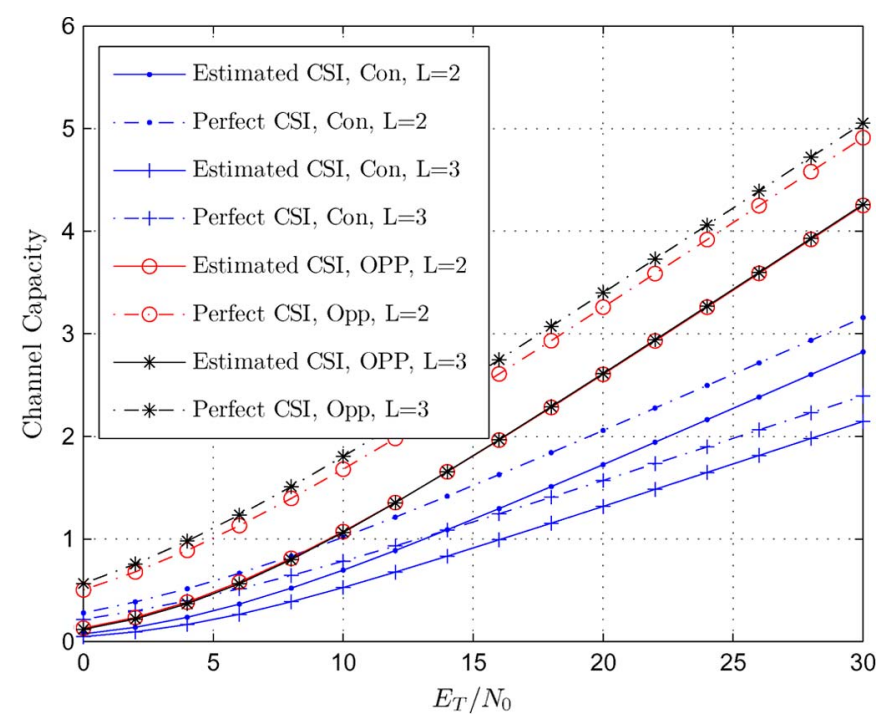

Fig. 5. Channel capacity for conventional and opportunistic relaying.

conventional counterpart for the same number of relays. It should be noted that, in the case of opportunistic relaying, as $L$ increases, the end-to-end SNR improves, and the average channel capacity of the opportunistic relaying increases. On the other hand, in conventional relaying, increasing $L$ has an opposing factor to SNR improvement, because it uses $L+1$ time slots and consequently reduces the channel capacity by a factor of $L+1$. The second factor has the dominant effect, which degrades the channel capacity performance with increasing number of relays, as observed in Fig. 5.

Finally, in Figs. 6 and 7, we investigate the effect of power allocation on the error rate performance. For conventional relaying, it is observed from Fig. 6 that optimized power allocation provides a performance improvement of $1.15 \mathrm{~dB}$ for $L=1$ at error probability of $10^{-4}$. The improvement decreases to 1 and $0.77 \mathrm{~dB}$ for $L=2$ and $L=3$, respectively. On the other hand, for opportunistic relaying, it is observed from Fig. 7 that the performance improvement is $1.15 \mathrm{~dB}$ for $L=1$. The 


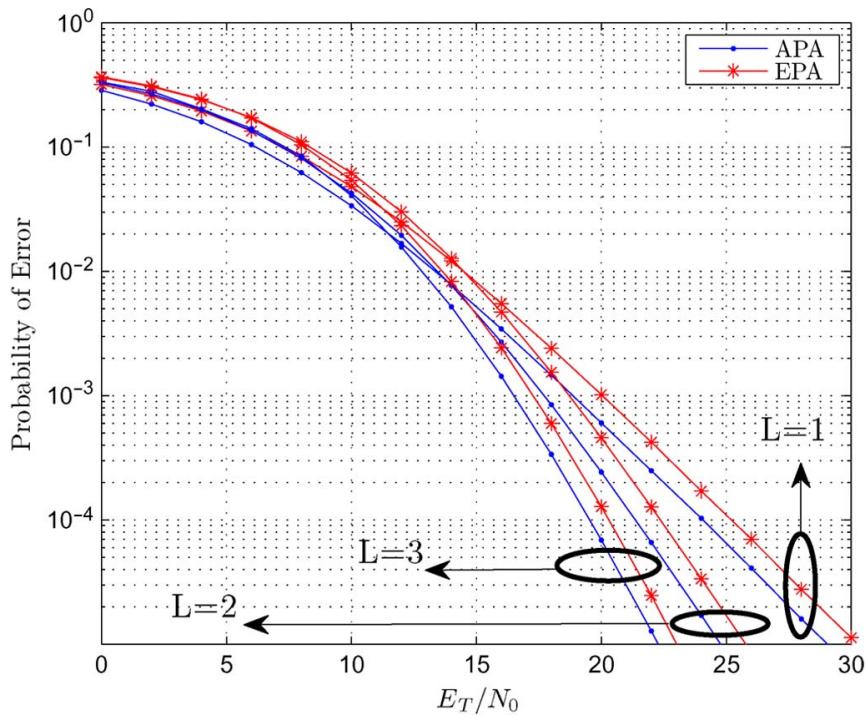

Fig. 6. Effect of power allocation on the performance of conventional relaying.

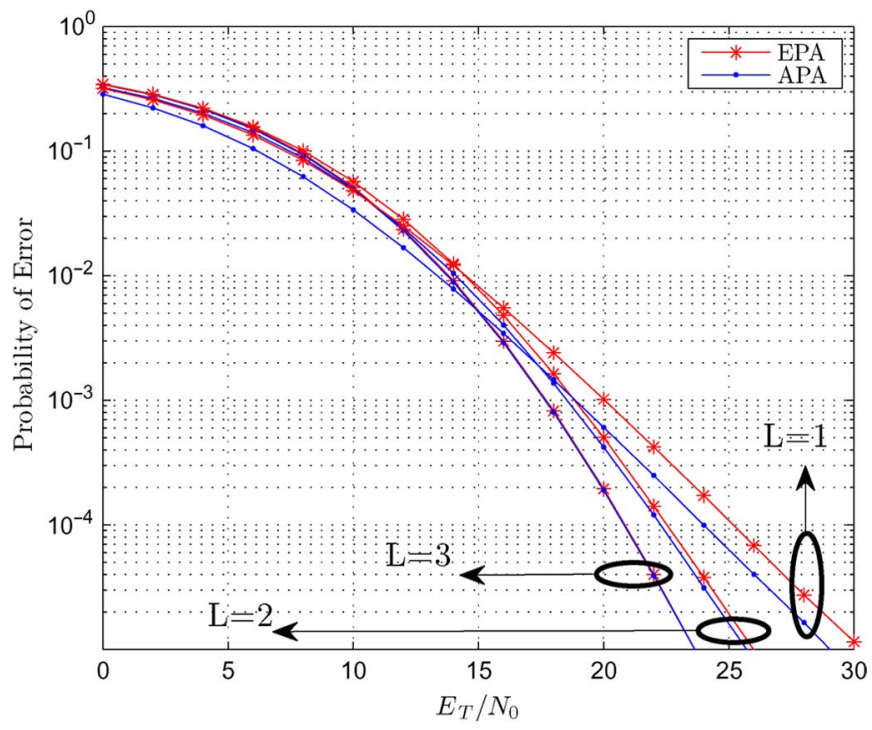

Fig. 7. Effect of power allocation on the performance of opportunistic relaying.

improvement gets smaller for increasing number of relays. It should be further emphasized that imperfect channel estimation reduces the improvement obtained through APA over equal power allocation (EPA) when compared with the perfect channel estimation case. For example, assume conventional relaying and $L=2$. For the perfect channel estimation case, APA brings a gain of $2.5 \mathrm{~dB}$ with respect to EPA. When imperfect channel estimation is considered, this gain reduces to $1 \mathrm{~dB}$. This is due to the fact that the imperfect estimation reduces the average received energy at both the relay and the destination nodes, i.e., $\hat{\gamma}_{S R_{i}}$ and $\hat{\gamma}_{R_{i}, D}$

\section{CONCLUSION}

In this paper, we have derived error probability, outage probability, and ergodic channel capacity expressions for a multirelay AF scheme in the presence of imperfect channel estimation. Utilizing a tight bound on the effective SNR, we have derived closed-form expressions for both conventional and opportunistic relaying. As demonstrated throughout this paper, our results generalize the existing results in the literature as to illustrate the effect of channel estimation errors. HighSNR analysis has also been made to provide insight into the achievable diversity orders. Derived results have been validated through extensive Monte Carlo simulation results. We have further used the derived error probability expressions to optimize the power allocation, which yields performance improvements of about $1 \mathrm{~dB}$.

\section{APPENDIX}

In this Appendix, we present the proof of (30). First note that the $n$th differentiation of $\gamma_{i}$ 's pdf at zero, $\left(\partial^{n} f_{\gamma_{i}} / \partial \gamma^{n}\right)(0)$, has a limited nonzero value. Using the chain rule and the fact that $\operatorname{Pr}\left[\gamma_{i} \leq 0\right]=0$, it can be shown that the pdf of the random variable $X=\max _{i \in L}\left(\gamma_{i}\right)$ can be written as

$$
\frac{\partial^{L-1} f_{X}}{d x^{L-1}}(0)=L \prod_{i=1}^{L} f_{\gamma_{i}}(0)
$$

where $f_{X}(x)$ is the pdf of the random variable $X$. Further, note that all the derivatives of the pdf of $\gamma_{\text {tot }}^{\text {Opp }}$, i.e., $f_{\gamma_{\text {opp }}}(\gamma)$, evaluated at zero up to order $L-1$ are zero, and the $L$ th-order derivative is given by

$$
\frac{\partial^{L} f_{\gamma_{\mathrm{tot}}^{\mathrm{Opp}}}}{\partial \gamma^{L}}(0)=f_{\gamma_{S D}}(0) \frac{\partial^{L-1} f_{X}}{\partial \gamma^{L-1}}(0) .
$$

Since we are integrating at the value around zero, the initial value theorem of Laplace transforms can be used to find (58). Noting that $\gamma_{\text {tot }}^{\text {Opp }}$ is the sum of two independent random variables, we have $M_{\gamma_{\text {top }}^{\text {Opp }}}(s)=M_{\gamma_{S D}}(S) M_{X}(s)$, where $M_{\gamma_{S D}}(s)$ and $M_{X}(s)$ are the Laplace transforms of $f_{\gamma_{S D}}(\gamma)$ and $f_{X}(\gamma)$, respectively. Using the initial value theorem, we obtain

$$
\frac{\partial^{L} f_{\gamma_{\mathrm{tot}}^{\mathrm{Opp}}}}{\partial \gamma^{L}}(0)=\lim _{s \rightarrow \infty} s^{L+1} M_{\gamma_{\mathrm{tot}}^{\mathrm{Opp}}}(s) \text {. }
$$

On the other hand, we have $\lim _{s \rightarrow \infty} s M_{\gamma_{S D}}(s)=f_{\gamma_{S D}}(0)$, which yields

$$
\lim _{s \rightarrow \infty} s^{L} M_{X}(s)=L \prod_{i=1}^{L} f_{\gamma_{i}}(0) .
$$

To find out the asymptotic behavior of the average error probability, we use the approximate expression given in [21]. Since the derivatives of $f_{\gamma_{\text {tot }}^{\text {Opp }}}(\gamma)$ up to the $k$ th order are null at $\gamma=0$, the approximate average error probability using the McLaurin series can be expressed as

$$
f_{\gamma_{\mathrm{tot}}^{\mathrm{Opp}}}(\gamma) \approx \frac{\prod_{i=1}^{k+1}(2 i-1)}{2(k+1) b^{k+1}} \frac{1}{k 1} \frac{\partial^{k} f_{\gamma_{\mathrm{tot}}^{\mathrm{Opp}}}}{\partial \gamma^{k}}(0)
$$

where $\partial^{k} f_{\gamma}(0) / \partial \gamma^{k}$ is the $k$ th-order derivative of the pdf, and the derivatives of $f_{\gamma}(\gamma)$ up to order $k+1$ are zero. Applying (57) and (61), the approximate error probability can be written as in (30). 


\section{REFERENCES}

[1] J. Laneman and G. Wornell, "Distributed space-time-coded protocols for exploiting cooperative diversity in wireless networks," IEEE Trans. Inf. Theory, vol. 49, no. 10, pp. 2415-2425, Oct. 2003.

[2] A. Sendonaris, E. Erkip, and B. Aazhang, "User cooperation diversity. Part I. System description," IEEE Trans. Commun., vol. 51, no. 11, pp. 1927-1938, Nov. 2003.

[3] R. Nabar, H. Bolcskei, and F. Kneubuhler, "Fading relay channels: Performance limits and space-time signal design," IEEE J. Sel. Areas Commun., vol. 22, no. 6, pp. 1099-1109, Aug. 2004.

[4] P. Mitran, N. Devroye, and V. Tarokh, "On compound channels with side information at the transmitter," IEEE Trans. Inf. Theory, vol. 52, no. 4, pp. 1745-1755, Apr. 2006.

[5] H. Ochiai, P. Mitran, and V. Tarokh, "Design and analysis of collaborative diversity protocols for wireless sensor networks," in Proc. 60th IEEE VTC, 2004, vol. 7, pp. 4645-4649.

[6] K. Azarian, H. El Gamal, and P. Schniter, "On the achievable diversitymultiplexing tradeoff in half-duplex cooperative channels," IEEE Trans. Inf. Theory, vol. 51, no. 12, pp. 4152-4172, Dec. 2005.

[7] A. Bletsas, A. Khisti, D. Reed, and A. Lippman, "A simple cooperative diversity method based on network path selection," IEEE J. Sel. Areas Commun., vol. 24, no. 3, pp. 659-672, Mar. 2006.

[8] H. Yomo and E. De Carvalho, "A CSI estimation method for wireless relay network," IEEE Commun. Lett., vol. 11, no. 6, pp. 480-482, Jun. 2007.

[9] F. Gao, T. Cui, and A. Nallanathan, "On channel estimation and optimal training design for amplify and forward relay networks," IEEE Trans. Wireless Commun., vol. 7, no. 5, pp. 1907-1916, May 2008.

[10] O. Amin, B. Gedik, and M. Uysal, "Channel estimation for amplifyand-forward relaying: Cascaded against disintegrated estimators," IET Commun., vol. 4, no. 10, pp. 1207-1216, Jul. 2010

[11] H. Mheidat and M. Uysal, "Non-coherent and mismatched-coherent receivers for distributed STBCs with amplify-and-forward relaying," IEEE Trans. Wireless Commun., vol. 6, no. 11, pp. 4060-4070, Nov. 2007.

[12] C. Patel and G. Stuber, "Channel estimation for amplify and forward relay based cooperation diversity systems," IEEE Trans. Wireless Commun., vol. 6, no. 6, pp. 2348-2356, Jun. 2007.

[13] Y. Wu and M. Patzold, "Performance analysis of cooperative communication systems with imperfect channel estimation," in Proc. IEEE ICC, Jun. 2009, pp. 1-6.

[14] B. Gedik and M. Uysal, "Impact of imperfect channel estimation on the performance of amplify-and-forward relaying," IEEE Trans. Wireless Commun., vol. 8, no. 3, pp. 1468-1479, Mar. 2009.

[15] S. Han, S. Ahn, E. Oh, and D. Hong, "Effect of channel-estimation error on BER performance in cooperative transmission," IEEE Trans. Veh. Technol., vol. 58, no. 4, pp. 2083-2088, May 2009.

[16] S. Kay, Fundamentals of Statistical Signal Processing: Estimation Theory. Englewood Cliffs, NJ: Prentice-Hall, 1993.

[17] P. Anghel and M. Kaveh, "Exact symbol error probability of a cooperative network in a Rayleigh-fading environment," IEEE Trans. Wireless Commun., vol. 3, no. 5, pp. 1416-1421, Sep. 2004.

[18] A. Papoulis and A. Maradudin, The Fourier Integral and Its Applications. New York: McGraw-Hill, 1963

[19] J. Proakis and M. Salehi, Digital Communications. New York: McGrawHill, 2001

[20] S. Ikki and M. Ahmed, "Performance of multiple-relay cooperative diversity systems with best relay selection over Rayleigh fading channels," EURASIP J. Adv. Signal Process., vol. 2008, pp. 1-7, Jan. 2008.

[21] Z. Wang and G. Giannakis, "A simple and general parameterization quantifying performance in fading channels," IEEE Trans. Commun., vol. 51, no. 8, pp. 1389-1398, Aug. 2003.

[22] A. Ribeiro, X. Cai, and G. Giannakis, "Symbol error probabilities for general cooperative links," IEEE Trans. Wireless Commun., vol. 4, no. 3, pp. 1264-1273, May 2005.

[23] M. Abramowitz and I. Stegun, Handbook of Mathematical Functions With Formulas, Graphs, and Mathematical Tables. New York: Dover, 1964.

[24] T. Nechiporenko, K. Phan, C. Tellambura, and H. Nguyen, "On the capacity of Rayleigh fading cooperative systems under adaptive transmission," IEEE Trans. Wireless Commun., vol. 8, no. 4, pp. 1626-1631, Apr. 2009.

[25] S. Ikki and M. Ahmed, "On the performance of amplify-and-forward cooperative diversity with the nth best-relay selection scheme," in Proc. IEEE ICC, 2009, pp. 1-6.

[26] S. Boyd and L. Vandenberghe, Convex Optimization. Cambridge, U.K.: Cambridge Univ. Press, 2004.

[27] J. Zhang, T. Zhang, J. Huang, and R. Yuan, "ABEP of amplify-andforward cooperation in Nakagami-m fading channels with arbitrary m," IEEE Trans. Wireless Commun., vol. 8, no. 9, pp. 4445-4449, Sep. 2009.
[28] Y. Zhao, R. Adve, and T. Lim, "Symbol error rate of selection amplifyand-forward relay systems," IEEE Commun. Lett., vol. 10, no. 11, pp. 757-759, Nov. 2006.

[29] R. Yuan, T. Zhang, J. Huang, J. Zhang, and Z. Feng, "Performance analysis of opportunistic cooperative communication over Nakagami-m fading channels," in Proc. IEEE Int. Conf. WCNIS, 2010, pp. 49-53.

[30] B. Maham and A. Hjorungnes, "Performance analysis of amplify-andforward opportunistic relaying in Rician fading," IEEE Signal Process. Lett., vol. 16, no. 8, pp. 643-646, Aug. 2009.

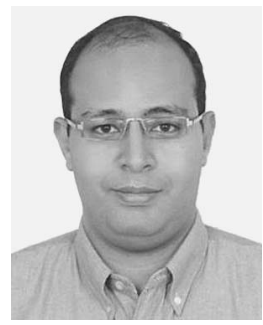

Osama Amin (S'07) received the B.Sc. degree in electrical and electronics engineering from South Valley University, Aswan, Egypt, in 2000, the M.Sc. degree in electrical engineering from Assiut University, Assiut, Egypt, in 2004, and the Ph.D. degree in electrical and computer engineering from University of Waterloo, Waterloo, ON, Canada.

$\mathrm{He}$ is currently a Postdoctoral Fellow with the Department of Electrical and Computer Engineering, University of British Columbia, Vancouver, BC, Canada. His research interests include cooperative communications, power line communication, adaptive systems, orthogonal frequency-division multiplexing, green communication, and channel estimation.

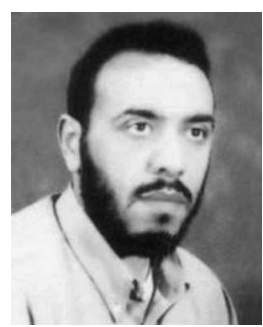

Salama Said Ikki (M'09) received the B.S. degree from Al-Isra University, Amman, Jordan, in 1996, the M.Sc. degree from The Arab Academy for Science and Technology and Maritime Transport, Alexandria, Egypt, in 2001, and the Ph.D. degree from Memorial University, St. John's, NL, Canada, in 2008, all in electrical engineering.

$\mathrm{He}$ is currently a Research Assistant with the Department of Electrical and Computer Engineering, University of Waterloo, Waterloo, ON, Canada. His research interests include communications and signal processing, specifically wireless cooperative networks, and multipleinput-multiple-output systems. His current research interests include signal processing, imperfect estimation, and cochannel interference for cooperative communications networks.

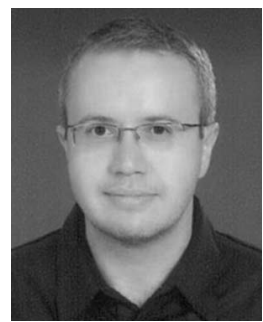

Murat Uysal (SM'07) was born in Istanbul, Turkey, in 1973. He received the B.Sc. and M.Sc. degrees in electronics and communication engineering from Istanbul Technical University, in 1995 and 1998, respectively, and the $\mathrm{Ph} . \mathrm{D}$. degree in electrical engineering from Texas A\&M University, College Station, in 2001

Since 2002, he has been with the Department of Electrical and Computer Engineering, University of Waterloo, Waterloo, ON, Canada, where he is currently an Associate Professor. He is currently on leave at Özyegin University, Istanbul, Turkey. He was a Guest Co-Editor for Wiley Journal on Wireless Communications and Mobile Computing Special Issue on "MIMO Communications" (October 2004). His research interests include communications theory and signal processing for communications, with special emphasis on wireless applications. His specific research interests include multiple-input-multiple-output communication techniques, space-time coding, diversity techniques and coding for fading channels, cooperative communication, and free-space optical communication.

Dr. Uysal is an Associate Editor for the IEEE TRANSACTIONS ON WIRELESS COMmunications and the IEEE COMMUNiCATIONS LeTters. He was a Guest Co-Editor for the IEEE Journal on SElected AREas IN Communications Special Issue on "Optical Wireless Communications" (December 2009). Over the years, he has served on the technical program committees of more than 60 international conferences in the communications area. He co-chaired the Communication Theory Symposium at the 2007 IEEE International Conference on Communications and the Communications and Networking Symposium at the 2008 Canadian Conference on Electrical and Computer Engineering. 